\title{
A Robust Neural Network Based Object Recognition System and Its SIMD Implementation
}

\author{
Alfredo Petrosino ${ }^{1}$ and Giuseppe Salvi ${ }^{2}$ \\ 1 INFM - University of Salerno \\ Via S. Allende, 84081 Baronissi (Salerno), ITALY \\ alfredo@synapse.irsip.na.cnr.it \\ 2 Istituto per la Ricerca sui Sistemi Informatici Paralleli, IRSIP-CNR \\ Via P. Castellino 111, 80131 Naples, ITALY
}

\begin{abstract}
Recognition of objects is a particularly demanding problem, if one considers that each image must be interpreted in milliseconds (usually 30 or 40 frames/second). In this paper we propose a massively parallel object recognition system, which makes use of the multi polygonal approximation scheme for the extraction of rotation and translation invariant shape features, in connection with artificial neural networks for the parallel classification of the extracted features. The system has been successfully applied for recognizing aircraft shapes in different sizes, orientations, with the addition of noise distortion and occlusion. Timings on the Connection Machine 200 are also reported.
\end{abstract}

\section{Introduction}

Recognition of shapes is a fundamental task in computer vision [14]. In the last decades, successful attempts have been made for the recognition of isolated objects with complete and well-defined boundaries. The usual process consists in the boundary extraction by applying a segmentation technique to the original grey scale image and then applying edge detectors and thinning algorithms to the binary image to retain the boundary width as small as possible. Once the boundary has been established the feature extraction task begins with extracting relevant features from each object present in the scene. The features are chosen so as to be invariant with respect to the object position, size and orientation.

In addition, many applications, including automatic target recognition (ATR), characterization of biomedical images, identification of industrial parts for product assembly, etc. require the recognition to take place in real-time and also in strongly noisy and occlusion conditions. This problem is very interesting if we consider a sequence of images with a resolution of $(512 \times 512)$ pixels at colours (8-24 bits for pixel) which are to be handled at a rate of 30 frames/second. The processing and the recognition of the contents of such images can be compared to the processing of 23.6 million of bytes/second. Thus, the use of more and more sophisticated parallel architectures and procedures of computation is 
natural and strongly needed, capable to deal with different data structures at the different levels of processing and interpretation. However, the use of parallel architectures gives rise to new problems concerning the choice of the modality of parallel computation (SIMD, MIMD) and the most efficient programming language. Current literature on parallel object recongition essentially deal with graph matching approaches implemented in parallel [1] on tree search algorithms [5], geometric hashing [2, 16] and parallel hypothesis generation [15]. Here we show that a complete set of computer vision tasks implementing filtering, feature extraction and statistic classification can be efficientely implemented as a whole system on a massively parallel SIMD architecture.

There are many techniques available to describe an object based on their boundaries (see for instance [4]). Among others, the polygonal approximation (specially that of high order) [10] combined with the Circular AutoRegressive (CAR) model approach, formerly proposed by Kashyap and Chellappa in 1981 [8], represents a good solution to handle the problems of occlusion, distorsion and noise, while remaining a low computational cost method. We shall use a modified version of the CAR model in combination with Artificial Neural Networks (ANNs) as nonparametric classifiers. The modifications introduced in the CAR model are based on the consideration of one set of predictive parameters is not sufficient to describe a shape, the model has to take into account more than one set of parameters, each corresponding to a different polygonal approximation. We shall refer to it as the Multi-Polygonal AutoRegressive Model (MPARM). In addition, Artificial Neural Networks (ANNs) are adopted due to their ability to adjust when given new information, neuronal massive parallelism typical of the SIMD parallel mode, fault tolerance to missing, confusing and/or noisy data. The paper is organized as follows. Section 2 briefly decribes the proposed approach whereas the third section reports an ensemble of experimental recognition results and times taken on the Connection Machine 200.

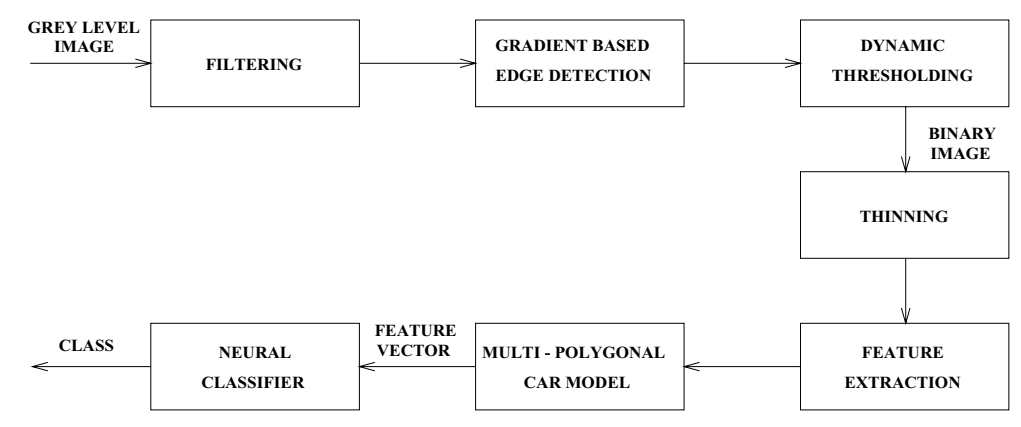

Fig. 1. The overall scheme of the object recognition system. 


\section{The Algorithm}

The overall system is depicted in Fig. 1 and the operations can be summarized as follows. The first stage is dedicated to detect the object boundary, after cleaning out the noise from the image by applying a linear smoothing filtering and a strongly noise independent segmentation procedure, based on the image entropy optimization between foreground and background [7]. The boundary is then coded by using elementary but salient features. We adopt and compare two strategies for the feature extraction : the first approach uses the variational angle sequence [9] (see Fig. 2(a))), while the second and more common approach uses the sequence of euclidean distances of points along the shape boundary from the centroid [3] (see Fig. 2] (b))).

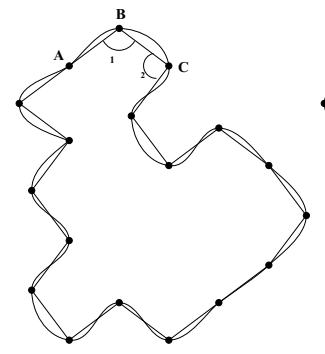

a)

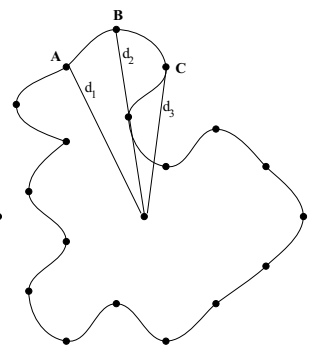

b)

Fig. 2. The adopted shape description models: angle of variation a) and distance from centroid b)

The sequence of shape features is modelled as a Circular Auto-Regressive (CAR) process [8], which is a parametric equation that expresses each sample of an ordered set of data samples as a linear combination of a specified number of previous samples plus an error term. Since the sequence is assumed to be circular, then it is invariant to rotation and translation. The form of the model is:

$$
y_{i}=\alpha_{0}+\sum_{k=1}^{m} \alpha_{k} y_{i-k} \quad \forall i=0,1, \ldots, n-1
$$

where $y_{i}$ is the current primary feature; $y_{i-k}$ is the feature detected $k$ times before the current features; $\alpha_{0}, \alpha_{1}, \alpha_{2}, \ldots, \alpha_{m}$ are the unknown CAR coefficients; $m$ is the model order. Let us indicate with $\beta=\left[\alpha_{0}, \alpha_{1}, \ldots, \alpha_{m}\right]$ the least square error (LSE) estimate of the CAR model. To improve the representativity of this solution the following method is adopted. If we fix $n$ as the number of line segments in the shape polygonal approximation, then $p=\lfloor(B / n)\rfloor$ pixels will lie on the contour between two end points, and $(p-1)$ polygonal approximations of the shape through model (2) are possible, depending from the starting point. 
The sequences of primary features generated for each of them may be slightly different. To overcome this problem we adopt an iterative scheme consisting of solving $p-1$ systems each having $n$ equations and $m+1$ unknown parameters. It is based on the consideration that the $p-1$ sequences are obtained in some order (clockwise or counterclockwise); firstly, the CAR vector for the first sequence of primary features is computed, then for two sequences, three sequences and so on. The process is repeated $p-1$ times, according to the number of sequences or polygonal approximations. In particular, denoted with $\beta_{j}$ the solution of the $j$-th system thus constructed, $j=1, \ldots, p-1$, let us define

$$
\varepsilon_{j}=\frac{\underline{\beta}_{j}^{T} \underline{\beta}_{j-1}}{\left\|\underline{\beta}_{j}\right\|^{2}\left\|\underline{\beta}_{j-1}\right\|^{2}} \quad j=2, \ldots p-1 \quad \text { and } \quad \varepsilon_{1}=\frac{1}{\left\|\underline{\beta}_{1}\right\|^{2}}
$$

a measure of similarity. The final feature vector $\beta^{*}$ is set to the solution of the $s$-th system, where $s=\arg \min _{0 \leq j \leq p-1}\left\{\varepsilon_{j}\right\}$. We shall refer to this way of proceeding as the Multi-Polygonal Auto-Regressive Model (MPARM). For sake of clearness, we denote in the following, as Scheme $I$ the MPARM applied to the variational angles, whereas Scheme $I I$ is the MPARM applied to euclidean distances for the centroid.

The sequences of CAR parameters are lastly fed into an Artificial Neural Network (ANN) trained to classify the planar objects with the Conjugate-Gradient (CG) algorithm [13. The process is repeated for the overall set of reference images. After learning, an unknown object passing through all the previous stages is classified as correct or not from the ANN with frozen weights.

The overall system described above has been implemented on a reconfigurable SIMD machine. The implemented algorithms were: convolution on a $3 D$ mesh, thresholding on a $2 D$ mesh, thinning and feature description on a $2 D$ mesh, conjugate gradient for neural learning on a $1 D$ mesh. We report in the next section the recognition performance and the parallel excecution times of the overal system.
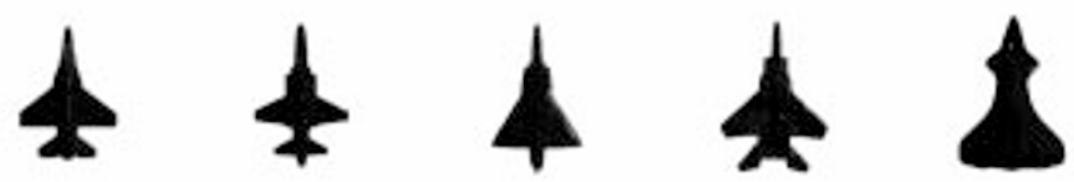

Fig. 3. Reference shapes. 


\section{$3 \quad$ Experimental Results}

\subsection{Recognition Performance}

The task faced was the recognition of five differently shaped aircrafts depicted in Fig. 2. The data set was formed by changing each aircraft in size, orientation and position. Specifically, the objects were $5^{0}$ rotated and translated in random positions within the image boundaries. The size of each aircraft was varied from 0.25 to 1.25 times the size of the original. By doing so, 5040 shapes were generated. For each shape the representative CAR vector was then extracted by using both feature angles of variation and euclidean distances from the centroid as elementary features.

The data set was consequently subdivided in 3600 shapes for the training and in 1440 shapes for the test of the neural classifiers.

We carried out various experiments with CAR models with different orders $m$. The value $m=20$ turned out to be optimal for our purposes. Table 1 shows the classification performance on the training and test sets for selected classifiers with different number of hidden units and shape description schemes.

\subsection{Recognition Results on Noisy and Occluded Objects}

Noise and distortion effects were introduced by adding random noise to the boundary points of 360 object shapes. To each contour pixel wass assigned a probability $p$ of retaining its original coordinates in the image plane and probability $q=(1-p)$ of being randomly assigned to the coordinates of one of its neighboring pixels. The degree of noise was augmented with increased values of the noise level $q$. We choose $q$ equal to $1 / 3$, i.e. one pixel over three was selected to change its own position. The set of noisy shapes was subdivided in 270 shapes for the training and in 90 shapes for the test of the neural classifiers.

A moderate amount of occlusion is also accommodated in our experiments. The occluded shapes have been obtained by cutting off a portion of the generated shapes with a straight line. The restriction on occlusion is that all the major portions, or branches, should remain; therefore, no major geometric property is changed. The set of occluded shapes has been subdivided in 540 shapes for the training and 180 shapes for the test of the neural classifiers. We tested the trained system over the set of noisy and occluded shapes. As expected, the classification performance obtained was substantially degraded. o get better classification performance we re-trained the previously neural classifiers with the 270 noisy shapes and 540 occluded shapes and, after convergence, they were tested on the 90 test noisy patterns and the 180 test occluded patterns. The results obtained are reported in Table 2 .

The best performance was achieved with 40 hidden neurons by using as elementary features both the angles of variation and the euclidean distances. 


\subsection{Timings}

The parallel algorithms were implemented in $\mathrm{C}^{*}$ language on the Connection Machine 200 (CM-200) 6], a fine-grain SIMD parallel computer configured with 8192 1-bit hypercube interconnected processors equipped with the Data Vault. We have configured the CM-200 to be used as a multi-dimensional mesh of processors.

As the computation progresses from one vision step to the next one, uniform partitioning is adopted. In the first stage of the shape recognition scheme (filtering, segmentation, edge extraction, thinning), the adopted re-mapping mode assures that performance gains are considerable, since at this stage there is no idle processor. This is not true for the second stage (feature extraction), where a contour following algorithm is involved, and the features are extracted only for the boundary pixels determined from the previous processing stage. There is of course evidence of a slowdown in performance, which is heavy in the context of the global performance reached. A MIMD solution should, in this case, give better performance. On the contrary, the expected performance gain due to the use of a neural classifier, where no idle processors are allowed, gives global efficient performance. Table 3 gives overall system performance from the first vision step, linear smoothing filtering, to the neural classification for the two schemes adopted for different image dimensions $(N=64,128,256)$.

A source of degradation in performance of the Scheme I against Scheme II is due to the need of calculating the trigonometric function involved in the computation of the angles of variation, which is slow on the Connection Machine-200. Commonly, a table lookup method is useful in computing an approximation to these functions. However, indexed addressing, which is required in lookup table, is as slow as indirect access on the Connection Machine-200, when each PE needs to access its table at a different address.

\section{Summary and Conclusions}

In this paper, a massively parallel system for the recognition of $2 D$ shapes by translation, scale and rotation invariant boundary representations is reported. The noise and occlusion immunity of the system has also been tested. The aim of this paper is born from the consideration that in the last decades a lot of computational vision algorithms have emerged as solution for designing efficient computer vision systems. Many of them are inherently sequential and results as regards the efficiency and applicability on parallel platforms are not reported. Others, parallel in nature, need to be tested on existing parallel platforms in order to fix their advantages and/or disadvantages when considered in a more complex vision framework. Thus, selection of efficient vision steps and their validation and testing on existing parallel platforms are mandatory and profitable for architecture designers. Our aim was to propose efficient parallel versions on SIMD machines of a selected set of algorithms very efficient from a qualitative point of view. Specifically, two methods for extracting primary features are employed and compared: angles of variation and euclidean distances from central 
moments were used. According to our results the scheme based on centroidal profile representation is more efficient in terms of recognition performance and the time required from the initial image capture from Data Vault to the display of the recognized object is approximately 0.3 seconds on a $8 \mathrm{k}$-processor Connection Machine running at $8 \mathrm{Mhz}$, for images of dimension up to $128 \times 128$ (VP ratio $=2$ ), strongly outperforming the timings reported in $[15$.

Table 1. The recognition performance by using Scheme I (a) and Scheme II (b).

\begin{tabular}{||c|c|c||}
\hline NET & TRAIN & TEST \\
\hline $20-5-5$ & 100.0 & 99.72 \\
\hline $20-10-5$ & 100.0 & 98.88 \\
\hline $20-15-5$ & 100.0 & 99.16 \\
\hline $20-20-5$ & 100.0 & 99.16 \\
\hline $20-25-5$ & 100.0 & 99.16 \\
\hline $20-30-5$ & 100.0 & 99.44 \\
\hline $20-35-5$ & 100.0 & 99.16 \\
\hline $20-40-5$ & 100.0 & 99.16 \\
\hline \multicolumn{3}{|c|}{ (a) }
\end{tabular}

(a)

\begin{tabular}{||c|c|c||}
\hline NET & TRAIN & TEST \\
\hline $20-5-5$ & 100.0 & 99.44 \\
\hline $20-10-5$ & 100.0 & 98.88 \\
\hline $20-15-5$ & 100.0 & 98.88 \\
\hline $20-20-5$ & 100.0 & 98.88 \\
\hline $20-25-5$ & 100.0 & 99.44 \\
\hline $20-30-5$ & 100.0 & 99.16 \\
\hline $20-35-5$ & 100.0 & 99.16 \\
\hline $20-40-5$ & 100.0 & 99.16 \\
\hline
\end{tabular}

(b)

Table 2. The recognition performance by using Scheme I (a) and Scheme II (b) for neural classifiers trained on occluded shapes.

\begin{tabular}{||c|c|c||}
\hline NET & TRAIN & TEST \\
\hline $20-5-5$ & 100.0 & 97.59 \\
\hline $20-10-5$ & 100.0 & 98.70 \\
\hline $20-15-5$ & 100.0 & 99.07 \\
\hline $20-20-5$ & 100.0 & 99.25 \\
\hline $20-25-5$ & 100.0 & 99.25 \\
\hline $20-30-5$ & 100.0 & 99.25 \\
\hline $20-35-5$ & 100.0 & 99.44 \\
\hline $20-40-5$ & 100.0 & 99.44 \\
\hline
\end{tabular}

(a)

\begin{tabular}{||c|c|c||}
\hline NET & TRAIN & TEST \\
\hline $20-5-5$ & 97.43 & 96.11 \\
\hline $20-10-5$ & 100.0 & 98.14 \\
\hline $20-15-5$ & 100.0 & 96.85 \\
\hline $20-20-5$ & 100.0 & 98.33 \\
\hline $20-25-5$ & 100.0 & 97.59 \\
\hline $20-30-5$ & 100.0 & 97.22 \\
\hline $20-35-5$ & 100.0 & 97.40 \\
\hline $20-40-5$ & 100.0 & 98.33 \\
\hline
\end{tabular}

(b)

Table 3. The experimental times (in seconds) for the whole process of computer vision as function of the size of the image $(N \times N)$.

\begin{tabular}{||c|c|c||}
\hline Recognition Scheme & I & II \\
\hline $\mathrm{N}=64($ VP ratio $=1)$ & 0.2595958 & 0.231238 \\
\hline $\mathrm{N}=128($ VP ratio $=2)$ & 0.384843 & 0.3357263 \\
\hline $\mathrm{N}=256($ VP ratio $=8)$ & 1.0183437 & 0.8930011 \\
\hline
\end{tabular}




\section{References}

[1] R. Allen, L. Cinque, S. Tanimoto, L. Shapiro, D. Yasuda, 'A Parallel algorithm for graph matching and its MasPar implementation', IEEE Trans. on Parallel and Dist. Systems, vol. 8, n. 5, pp. 490-500, (1997).

[2] O. Bourdon, G. Medioni, 'Object Recognition Using Geometric Hashing on the Connection Machine', Proc. of Intern. Conf. on Pattern Recognition, pp. 596-600, 1988.

[3] C. C. Chang, S. M. Hwang, D. J. Buehrer, 'A shape recognition scheme based on the relative distances of feature points from the centroid', Pattern Recognition, 24, No. 11, pp. 1053-1063, (1991).

[4] R. C. Gonzalez, P. Wintz, Digital Image Processing, Reading, MA, Addison-Wesley (1977).

[5] J. G. Harris, A. M. Flynn, 'Object recognition using the Connection Machine's router', Proc. of Computer Vision and Pattern Recognition, pp. 134-139, (1986).

[6] W. D. Hillis, The Connection Machine, MIT Press, Cambridge, MA, 1985.

[7] J. N. Kapur, P. K. Sahoo, 'A new method for grey level image thresholding using the entropy of the histogram', Computer Vision, Graphics and Image Processing, 29, (1985).

[8] R. Kashyap, R. Chellapa, 'Stochastic models for closed boundary analysis: representation and reconstruction', IEEE Trans. Inf. Theory, 27, 5, pp. 109-119 (1981).

[9] N. R. Pal, P. Pal, A. K. Basu, 'A new shape representation scheme and its application to shape discrimination using a neural network', Pattern Recognition, 26, 4, pp. 543-551 (1993).

[10] T. Pavlidis, 'Algorithms for shape analysis of contour and waveforms', IEEE Trans. Patt. Anal. Machine Intell., 2, 4, pp. 301-312 (1980).

[11] A. Petrosino, G. Salvi, 'A two subcycle parallel thinning algorithm and its parallel implementation on SIMD machines', IEEE Trans. on Image Processing, to appear, (1999).

[12] A. Petrosino, G. Salvi, 'Recognizing planar objects with neural networks', Proceedings of Intern. Conf. on Neural Networks ICANN '94, M. Marinaro and P. Morasso eds., pp. 835-838, Springer Verlag (1994).

[13] M. J. D. Powell, 'Restart procedures for the Conjugate Gradient method', Mathematical Programming, 12, pp. 241-254 (1977).

[14] P. Suetens, P. Fua, A. J. Hanson, 'Computational strategies for object recognition', ACM Computing Surveys, 24, 1, (1992).

[15] L. W. Tucker, C. R. Feynman, D. M. Fritzsche, 'Object recognition using the Connection Machine', Proc. IEEE Conf. Computer Vision and Pattern Recognition, June 1988, pp. 871-877.

[16] C.-L. Wang, V. K. Prasanna, H. J. Kim, A. A. Khokhar, 'Scalable Data Parallel Implementations of Object Recognition Using Geometric Hashing', Journal of Parallel and Distributed Computing, 1994. 